\title{
Hydrogen-Induced Changes in Palladium Surface Structure: Field Ion Microscopy Study
}

\author{
A. TomaszewsKa* AND Z.M. STȨPIEŃ \\ Institute of Physics, Jan Długosz University \\ al. Armii Krajowej 13/15, 42-200 Czȩstochowa, Poland
}

\begin{abstract}
In this work the field ion microscopy technique was used in order to examine hydrogen-induced changes in palladium surface structure in the context of surface palladium hydrides formation. The goal of the study is to determine the crystallographic planes on which the particles of interest are formed with greatest intensity. Employing a method which consists in finding the differences in the brightness of field ion microscopy images we found that the structures of palladium surface hydrides, the size of which varies from 1 to $30 \mathrm{~nm}$, develop mainly on $\{110\}$ planes of palladium.
\end{abstract}

PACS numbers: 68.37.-d, 68.37.Vj, 79.70.+q

\section{Introduction}

The hydrogen-palladium system, because of its technological importance resulting from its unique adsorption, absorption and permeability properties, has been for many years the object of both experimental and theoretical studies carried out employing a wide variety of methods. The experimental works, performed by using techniques directed strictly towards surface investigations, reveal the occurrence of small well-ordered patches, separated by steps, dislocations, facets and grain boundaries on the metal surface exposed to hydrogen [1]. On the other hand, in the case of the system of interest, phenomena which lead to the changes of substrate atom positions may appear. The process of surface reconstruction, which in the case of the $\mathrm{Pd} / \mathrm{H}$ system takes place on the (110) plane at coverage exceeding 1.0 ML [1-4], has been listed as one of the most spectacular effects leading to this kind of changes. Also, if the hydrogen-promoted changes in substrate structure are taken into account, the formation of surface chemical compounds including metal surface hydrides cannot be omitted.

*corresponding author; e-mail: a.tomaszewska@ajd.czest.pl 
According to the definition, $d$-transition-metal hydrides are considered as solid compounds in which hydrogen atoms occupy interstitial positions inside bulk metal [5]. It has to be noticed that hydrogen atoms are able to enter an interstice if the following conditions are fulfilled. First, the space available to hydrogen atoms must exceed $0.37 \AA$ (Westlake's criterion) [6]. Second, the distance between hydrogen atoms occupying the neighboring voids must be greater than $2.1 \AA$ (Switendick's criterion) [7]. In the case of a metal of fcc structure the occupation of all accessible tetrahedral and octahedral interstices corresponds to the formation of $\mathrm{MeH}_{3}$ phase (here Me relates to the atom of the metal). Although the formation of compounds of such stoichiometry is theoretically likely, in reality however, under critical temperature and pressure conditions, the formation of $\mathrm{PdH}_{x}$ with $x$ not exceeding unity was observed. On the other hand, in the presence of an external electric field the formation of surface compounds of $\mathrm{PdH}_{x}(x=1,2,3,4)$ was reported $[8,9]$.

In our previous paper [10] the role played by the external electric field of a magnitude of $10-40 \mathrm{~V} / \mathrm{nm}$ in the hydrogen-induced alterations in palladium surface structure, in particular, in the efficiency of palladium hydrides formation, was examined. However, any effort has not been made in order to identify those crystallographic planes on which the strongest changes of interest appear.

In the present investigation field ion microscopy (FIM) has been employed in order to study changes in palladium surface structure exposed to hydrogen under the pressure of $10^{-4} \mathrm{hPa}$. The useful benefit of this technique consists in the possibility of observing changes that appear on different surface regions of the specimen at any time. The main goal of this study is to find out the regions on which the most pronounced alterations are induced. For this purpose a method of processing the FIM image, whose details are described briefly in the next section, is proposed.

\section{Experimental}

A conventional metal FIM apparatus equipped with a standard ultrahigh vacuum system as well as low-pressure measurement facilities was used. The images were obtained, using krypton as the image gas, via an internal channel plate intensifier and recorded with a CCD camera. During the course of imaging the specimen was kept at liquid nitrogen temperature.

The field ion specimen was prepared from a $0.1 \mathrm{~mm}$ palladium wire of $99.99 \%$ purity by means of ac $(\approx 5 \mathrm{~V})$ electropolishing in $38 \%$ solution of $\mathrm{HCl}$ in glycerine [11]. Before experiment, the specimen surface was shaped to an atomically smooth end-form by using both annealing at $1000 \mathrm{~K}$ and field evaporation. To observe hydrogen-promoted changes in palladium surface structure on the atomic scale the following procedure was applied. First, after evacuating the system to the background pressure of $1 \times 10^{-9} \mathrm{hPa}$, krypton image gas at a pressure of $10^{-3}-5 \times 10^{-4} \mathrm{hPa}$ was introduced into the chamber and a set of clean palladium 
surface patterns were recorded. Next, the system was pumped out again to the background pressure and the palladium surface was exposed to hydrogen under the pressure $4.5 \times 10^{-6}-2.5 \times 10^{-4} \mathrm{hPa}$. As soon as the images of palladium surface interacting with hydrogen were taken, the system was evacuated again and eventually krypton at a pressure of $10^{-4} \mathrm{hPa}$ was admitted to the system. The images taken under experimental conditions as described above are expected to show the topographic changes in surface structure caused by the interaction of the surface with hydrogen in the presence of an external electric field.

In order to identify the regions of the specimen on which the most pronounced changes have occurred, a procedure is proposed, which consists in finding the absolute differences in brightness of FIM images taken before and after metal surface exposure to hydrogen at the same imaging voltage. In particular, the FIM image spots present on the pattern of the clean surface of palladium are to be removed from the FIM pattern of the hydrogen-exposed palladium surface. Then, the spots that remain on the resultant pattern are believed to correspond to palladium atoms whose equilibrium positions were changed under the influence of palladium-hydrogen interaction.

\section{Results and discussion}

By means of the imaging procedure described in the foregoing section, three series of FIM images of the palladium surface were obtained. A sequence of the micrographs taken at the same imaging voltage is shown in Fig. 1. By comparing the micrographs with the stereographic projection of the (001)-oriented fcc lattice, the major crystallographic planes can be indicated [12]. On the clean palladium surface (Fig. 1a) a significant thermal disorder is noticed, which is due to pre-annealing the specimen as well as to field evaporation. However, the fourfold symmetry features, typical of this lattice structure, are clearly seen. Besides, the developed edges of several planes give evidence for surface rearrangement. The images taken after palladium exposure to hydrogen are characterized by the absence of developed edges (Fig. 1b). In addition, a great deal of bright spots' islands is noticeable. These regions are the ones where the processes of field ionization and field adsorption take place with a great efficiency. This is in particular the case of $\{110\}$ surfaces which are known to have quite a rough structure. As it was mentioned in the foregoing section, we believe that upon analyzing patterns taken after palladium exposure to hydrogen (Fig. 1c) the adsorbate-induced alterations in substrate surface structure may be identified. Our belief is based on the difference in ionization potential between hydrogen and krypton. Owing to the fact that the value of the ionization potential of krypton is lower than that of hydrogen [11] we can expect that the surface complexes formed in the course of palladium exposure to hydrogen do not undergo desorption during krypton-FIM imaging.

The picture resulting from applying the procedure of searching differences in contrast between FIM images is shown in Fig. 1d. It is clearly seen from this 

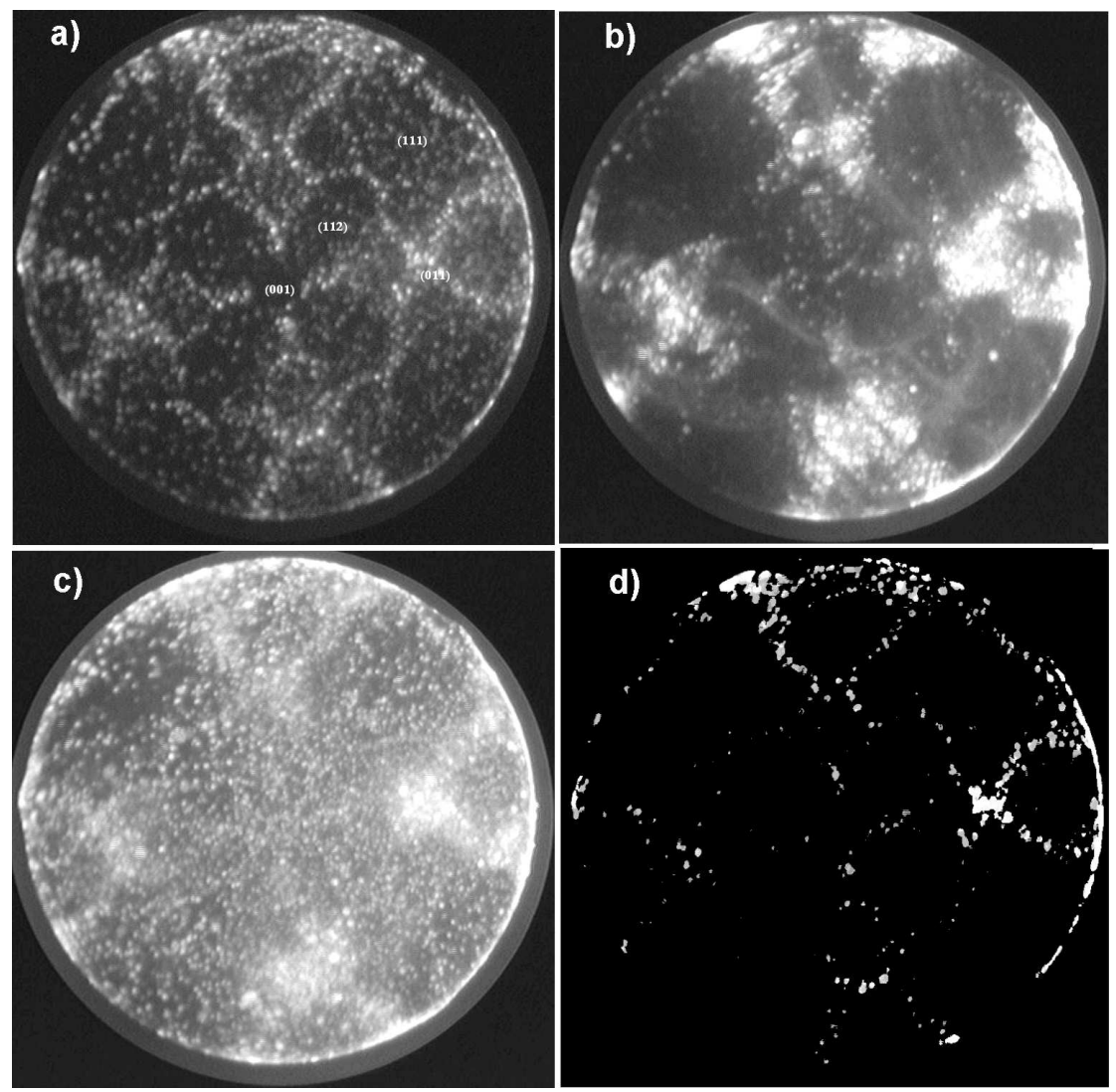

Fig. 1. A sequence of palladium FIM images of the palladium surface. The imaging voltage was $27.1 \mathrm{kV}$. (a) Image of the clean surface, obtained with krypton image gas. (b) Image of the clean surface, obtained with hydrogen image gas. (c) An image taken after surface exposure to hydrogen obtained with krypton image gas. (d) A picture of differences in brightness of patterns (c) and (a).

that the changes in palladium surface structure that accompany the interaction of hydrogen with the highly diversified surface of the field-emitter tip of palladium in the presence of an external electric field occur mostly on the $\{110\}$ plane. The size of spots that correspond to these changes was estimated to vary from 1 to $30 \mathrm{~nm}$.

For several reasons, the appearance of the most pronounced hydrogen-induced structural alterations just on the $\operatorname{Pd}\{110\}$ is not surprising. First, the planes of this type affected by the presence of hydrogen species undergo the process of surface reconstruction accompanied by the lattice changes by about $\pm 0.1 a[13]$. Second, the rough structure of such planes is the reason why the magnitude of the local electric field existing there is higher than on other regions of the emitter tip. Hence, the processes of field adsorption of hydrogen followed by adsorbate diffu- 
sion into the subsurface voids and consequently the formation of surface palladium hydrides take place there most intensively.

In paper [10] we showed that the formation of surface palladium hydrides is the most prominent reason that accounts for increase in interatomic distances on the palladium surface of the emitter tip influenced by the presence of hydrogen adsorption species. The changes in $\mathrm{Pd}-\mathrm{Pd}$ distance were investigated as a function of electric field magnitude.

Indeed, at the temperature close to that in our experiment, under zero-field conditions, a strong adsorption of hydrogen on $\mathrm{Pd}$ has been observed [14-16] without hydrogen desorption during evacuation of the cell. The equilibrium pressure for $\mathrm{PdH}$ formation at the temperature $85 \mathrm{~K}$ is of the order of $10^{-5} \mathrm{hPa}$. Hence, if the kinetic barrier did not hinder the hydride formation, the hypothesis of $\mathrm{PdH}$ formation under our experimental conditions $\left(p=2.5 \times 10^{-4} \mathrm{hPa}\right)$ seems to be reasonable.

Furthermore, thermal desorption results from the Pd (110) surface exposed to hydrogen [3] are a strong evidence for formation of surface hydrides at low temperature.

We found previously that in the field of strength exceeding $23 \mathrm{~V} / \mathrm{nm}$ palladium hydrides are formed with the greatest efficiency. Currently, on the basis of considerations presented here we are able to complement that conclusion by the indication of the $\{110\}$ crystallographic planes as the ones on which the formation of surface hydrides is most pronounced.

Summarizing, it has been shown in this paper that the formation of hydride structures of nanometer size on the palladium surface is possible by using the FIM technique. In our opinion, some investigations of the magnetic properties of surface palladium hydrides should be undertaken as the next step towards better understanding the specificity of palladium-hydrogen interaction in the presence of the high external electric field.

\section{Conclusions}

We have used the FIM approach to study the structural changes that appear on a palladium surface exposed to hydrogen. It is determined by employing the used technique of image processing, which consists in finding the differences in brightness of patterns of the surface influenced and that one not affected by hydrogen adsorption species, that the most pronounced changes of interest appear on the planes of $\{110\}$ type. The process of surface hydride formation is believed to be mostly responsible for the appearance of the observed changes.

The possibility of formation of nanometer-size objects on a metal surface seems to open a promising opportunity for applying this kind of compounds in nanotechnology. However, further effort directed towards the investigation of magnetic properties of this adsorption system is necessary. 


\section{Acknowledgments}

The authors are greatly indebted to Professor A. Ciszewski from the University of Wrocław for giving one of the authors (A.T.) the opportunity to carry out the FIM experiment in his laboratory.

The authors acknowledge the financial support of the Ministry of Science and Higher Education (grant No. N207 017 31/0864).

\section{References}

[1] K. Christmann, Surf. Sci. Rep. 9, 1 (1988).

[2] M.G. Cattania, V. Penka, R.J. Behm, K. Christmann, G. Ertl, Surf. Sci. 126, $382(1983)$

[3] J.W. He, D.A. Harrington, K. Griffiths, P.R. Norton, Surf. Sci. 198, 413 (1988).

[4] J. Yoshinobu, H. Tanaka, M. Kawai, Phys. Rev. B 51, 4529 (1995).

[5] A.F. Wells, Structural Inorganic Chemistry, Oxford Univ. Press, New York 1991.

[6] D.G. Westlake, J. Less-Common Met. 91, 275 (1983).

[7] A.C. Switendick, Z. Phys. Chem. N.F. 117, 89 (1979).

[8] S. Kapur, E.W. Müller, Surf. Sci. 66, 45 (1977).

[9] Z.M. Stępień, T.T. Tsong, Surf. Sci. 409, 57 (1998).

[10] A. Tomaszewska, A. Ciszewski, Z.M. Stẹpień, Appl. Surf. Sci. 254, 4386 (2008).

[11] A.J. Nam, A. Teren, T.A. Lusby, A.J. Melmed, J. Vac. Sci. Technol. B 13, 1556 (1995).

[12] E.W. Müller, T.T. Tsong, Field Ion Microscopy: Principles and Applications, American Elsevier Publ. Co., New York 1969.

[13] H. Niehus, C. Hiller, G. Comsa, Surf. Sci. 173, L 599 (1986).

[14] R. Duś, Surf. Sci. 42, 324 (1973).

[15] R. Duś, Surf. Sci. 52, 440 (1975).

[16] R. Duś, E. Nowicka, Z. Wolfram, Surf. Sci. 269/270, 545 (1992). 